\title{
BMJ Open Clinico-epidemiological characteristics of Kawasaki-like disease in paediatric patients with COVID-19: a protocol for rapid living systematic review
}

\author{
Abhinav Sinha (D) , ${ }^{1}$ Swetalina Nayak, ${ }^{1}$ Priyadarshini Dehuri, ${ }^{2}$ Srikanta Kanungo, ${ }^{1}$ \\ Sanghamitra Pati ${ }^{1}$
}

To cite: Sinha A, Nayak S, Dehuri $\mathrm{P}$, et al. Clinicoepidemiological characteristics of Kawasaki-like disease in paediatric patients with COVID-19: a protocol for rapid living systematic review. BMJ Open 2020;10:e041160. doi:10.1136/ bmjopen-2020-041160

- Prepublication history and supplemental material for this paper is available online. To view these files, please visit the journal online (http://dx.doi. org/10.1136/bmjopen-2020041160).

Received 01 June 2020 Revised 26 November 2020 Accepted 07 December 2020

Check for updates

(C) Author(s) (or their employer(s)) 2020. Re-use permitted under CC BY-NC. No commercial re-use. See rights and permissions. Published by BMJ.

${ }^{1}$ ICMR-Regional Medical Research Centre, Bhubaneswar, Odisha, India

${ }^{2}$ Department of Pathology, Institute of Medical

Sciences and SUM Hospital, Bhubaneswar, Odisha, India

Correspondence to Dr Sanghamitra Pati; drsanghamitra12@gmail.com and

Dr Srikanta Kanungo; srikantak109@gmail.com

\section{ABSTRACT}

Introduction The COVID-19 outbreak has posed a major challenge to healthcare providers. Due to its communicable nature, very stringent public health interventions have been put in place worldwide; yet, it still poses new emerging challenges, one of the most recent being a multisystem inflammatory condition with clinical features resembling Kawasaki-like disease and toxic shock syndrome in children and adolescents. The data on this novel condition are scarce which need to be reported to identify its clinico-epidemiological and geographical distribution. There is an urgent need to generate evidence for diagnosis and management of this condition in the midst of a pandemic.

Methods and analysis This systematic review will be conducted using Medline database searched through PubMed, Embase, Ovid; and Google Scholar, ProQuest and EBSCO databases will also be searched along with grey literature with the aim to identify the clinical features, aetiopathology, laboratory findings, treatment modes and outcomes of Kawasaki-like disease among paediatric patients suffering from COVID-19. Original articles reporting Kawasaki-like disease in paediatric patients with COVID-19 will be retrieved after screening by two independent reviewers. Data will be extracted in a specially designed form and studies will be assessed independently for risk of bias. Data will be extracted for the following: author, journal title, publication year, study design, study setting, demographic characteristics, sample size, clinical features, aetiopathology, laboratory findings, modes and doses of treatment given, strength and weakness of studies. A descriptive and quantitative analysis will be completed.

Ethics and dissemination This is a literature-based review study with no ethical concerns. We will publish the results in a peer-reviewed journal and present at a conference.

PROSPERO registration number CRD42020187427.

\section{INTRODUCTION}

The COVID-19 outbreak caused by SARS-CoV-2 has posed a major challenge to healthcare providers. ${ }^{12}$ Due to the communicable nature of this virus, very stringent public health interventions have been put in

\section{Strengths and limitations of this study}

- A comprehensive assessment of clinicoepidemiological features of a novel condition will be done in this study.

- This systematic review will inform healthcare providers about this novel condition and ways to manage it.

- During the primary screening phase, reviewers will be blinded to minimise selection bias.

- Data extraction and quality assessment will be performed by independent reviewers, thus minimising bias and maintaining quality.

place worldwide. ${ }^{3}{ }^{4}$ Yet, it strives to pose new emerging challenges. One of the recent being multisystem inflammatory syndrome with clinical manifestations resembling Kawasakilike disease and toxic shock syndrome in children and adolescents. ${ }^{56}$ Although it has been observed that older adults with underlying comorbidities are vulnerable to a more severe form of the disease which may require intensive care support, some children are also hospitalised. ${ }^{78}$

Recently, clusters of children and adolescents from Europe and North America have reportedly been admitted to intensive care units (ICUs) with a multisystem inflammatory syndrome with clinical features resembling Kawasaki-like disease and toxic shock syndrome. ${ }^{910}$ This condition has temporarily been associated with COVID-19 as majority of the cases have shown positive serology in the laboratory investigation. ${ }^{11} 12$ The full range and geographical distribution of the disease is yet not clear owing to the probability of not being recognised in other parts of the world. In order to generate evidence, there should be a standard data collection technique reporting clinical presentation, severity, outcomes and epidemiology throughout the 
globe. It is important to understand the causes and risk factors of the condition so that evidence-based management can be described.

A preliminary case definition has been given by the WHO based on both clinical features, as well as laboratory investigations, which can be revised in future depending on the availability of more data. The present definition ${ }^{13}$ states that any child or adolescent of 0-19 years of age, who has fever for 3 or more days along with any two of the five following laid criteria which include:

1. Mucocutaneous inflammation which can be oral or on limbs, or rash, or non-purulent conjunctivitis.

2. Hypotension or shock.

3. Clinical features of cardiac involvement like pericarditis, coronary abnormalities, valvulitis or myocardial dysfunction (including findings of echocardiography or higher levels of troponin/N-terminal pro b-type natriuretic peptide).

4. Confirmed coagulopathy (evident by prothrombin time, partial thromboplastin time and increased Ddimers).

5. Having acute gastrointestinal symptoms like vomiting, diarrhoea or abdominal pain.

This can be accompanied with raised levels of erythrocyte sedimentation rate, $\mathrm{C}$ reactive protein or prolactin marking inflammation. Also, there should not be any other causes of microbial inflammation which includes bacterial sepsis and staphylococcal or streptococcal shock syndromes. All these criteria should also be accompanied by the presence of COVID-19 infection (confirmed by reverse transcription-PCR (RT-PCR), antigen test or serology positive) or the case be a likely contact of a patient with COVID-19.

This case definition will help in identifying and treating the cases, and at the same time will assist in surveillance.

In the midst of a pandemic, there is an urgent need to collect evidence for diagnosis and management of this new challenge in the form of a syndrome associated with paediatric patients with COVID-19. Hence, in order to generate the most up-to-date evidence, while maintaining scientific rigour and quality, a systematic review of clinico-epidemiological characteristics of this syndrome is needed. Additionally, studies relevant for these research questions will likely be continuously published in the foreseeable future. Moreover, traditional systematic reviews risk becoming rapidly outdated when new evidence is published almost on a daily basis, and it is not an option to wait until the pandemic is over to publish a systematic review on the full body of evidence. Hence, a rapid systematic review that is continuously updated (living) for this syndrome is necessary. With this background, we present the protocol for this systematic review with the following objectives.

\section{OBJECTIVES}

To present a protocol for rapid living systematic review with the following research questions in mind:
1. What are the clinical and epidemiological features, the aetiopathology, the measures of laboratory findings and their variability, and the treatment modes and doses used among the paediatric patients suffering from COVID-19 along with symptoms of Kawasaki-like disease?

2. What are the outcomes of paediatric patients suffering from COVID-19 and having Kawasaki-like disease?

\section{METHODS AND ANALYSIS}

\section{Standards}

This study protocol was developed following Preferred Reporting Items for Systematic Review and Meta-Analysis Protocols (PRISMA-P) (online supplemental file 3) reporting guidelines. ${ }^{14}$ This systematic review on clinicoepidemiological characteristics of Kawasaki-like disease in paediatric patients with COVID-19 will be performed and reported following PRISMA guidelines.

\section{Protocol registration}

The protocol for present study is registered with the International Prospective Register of Systematic Reviews. ${ }^{15}$ In due course of the study, if any change in the protocol will be made, it will be updated here.

\section{Eligibility criteria}

Study characteristics/design

We will include observational studies for this systematic review.

Inclusion:

1. Original articles reporting observational studies including case report, case series, cross-sectional, casecontrol and cohort study.

2. Articles reporting Kawasaki-like disease in children and adolescents suffering from COVID-19.

3. Kawasaki-like disease symptoms, if not explicitly stated by the authors of included studies, will be inferred by the reviewer.

Exclusion:

1. Dissertations, conference proceeding and reviews will be excluded.

2. Studies with no accessible full-text versions will also be excluded.

3. In vitro and animal studies will not be considered.

\section{Types of participants/population}

This study proposes to target paediatric patients with COVID-19 exhibiting Kawasaki-like disease.

Inclusion:

1. Children and adolescent patients with COVID-19, aged $0-19$ years.

2. With Kawasaki-like disease symptoms.

3. Included cases of COVID-19 infection should be confirmed either by RT-PCR or antigen test or serology positive or the case be a likely contact of a patient with COVID-19. 


\section{Setting and time frame}

This systematic review will cover all studies conducted in hospital or clinical settings including special hospital set-up for COVID-19. Articles will be screened from 31st December 2019 when the initial case of COVID-19 outbreak was reported from China until 30th September 2020 in order to generate rapid evidence. This review will be living in nature, being updated every 2 months from first publication until May 2021, at which point the need for further updates, and their regularity, will be reconsidered.

\section{Report characteristics}

Published articles/reports along with preprint versions of the articles will be reviewed. There will be no limitation for language and date of acceptance or publication.

\section{Information sources}

For this systematic review, electronic databases, websites of international organisations like the WHO, grey literature including reports and researches will form the source of information. A comprehensive search will be done using electronic databases, such as Medline, EBSCO and ProQuest. PubMed and Google Scholar search engines will be used to retrieve studies. Additionally, Medline will be covered by searching Embase and Ovid databases. PubMed will be used to design search strategy using Medical Subject Headings (MeSH) terms and associated keywords. Other frequently used and popular phrases from the existing literature will be used to make the search comprehensive and exhaustive. The same search strategy will also be used for other databases.

Additionally, COVID-19-specific databases (which include preprint repositories) from December 2019 to the current date will also be searched. Preprint repositories to be included are medRxiv (https://www.medrxiv. org/) and bioRxiv (https://www.biorxiv.org/) . The EPPI Centre living systematic map of the evidence (http:// eppi.ioe.ac.uk/cms/Projects/DepartmentofHealthan dSocialCare/Publishedreviews/COVID- 19Livingsystematicmapoftheevidence/tabid/3765/Default.aspx) and the COVID-19 Research Database maintained by the WHO (https://www.who.int/emergencies/diseases/ novel-coronavirus-2019/global-research-on-novel-coronavirus-2019-ncov) will also be a source of information for this study.

Also, the reference lists of the included articles will be considered as a source of information which will be hand searched.

\section{Search strategy}

The basic search syntax will consist of three concepts: Kawasaki disease, COVID-19 and Pediatric population. The MeSH terms used for Kawasaki disease will be 'Multisystem inflammatory Syndrome', 'Pediatric multisystem inflammatory disease', 'Hyperinflammatory shock', 'Toxic shock syndrome', 'kawasaki disease', Vasculitis, 'pediatric multisystem inflammatory disease, COVID-19 related'; for COVID-19 it will be 'COVID-19' [Supplementary Concept] and for pediatric population it will consist of 'Child'.

All the three concepts will be added using AND, that is, \#1 AND \#2 AND \#3.

The detailed search strategy is available in the online supplemental file 1 .

\section{Study records}

\section{Selection process}

In the first phase, titles and abstracts will be screened for potential eligibility by two independent reviewers. During this phase, the reviewers will be blinded to the study details like authors, journal or year of publication to minimise selection bias. The selected articles will further be categorised as relevant, irrelevant and unsure. Any article categorised under irrelevant by both the reviewers will be eliminated. In the second phase, full texts of potentially eligible articles based on the first phase of screening will be obtained. These full-text articles will undergo another round of screening based on inclusion and exclusion criteria by two other reviewers. Any dissent over the sieving of the full-texts articles will be resolved by the entire team in consensus.

\section{Data management}

A preformed data extraction sheet will be used to extract and enter data by two reviewers independently. These two sheets will be independently assessed by a third reviewer, and compared and checked for disparities. Any potential differences arising will be discussed and resolved by all three of the former reviewers. If not resolved, the entire team would be contacted to resolve matter in consensus. If any data in the article are found missing, incomplete or unclear, relevant authors will be enquired for the same through email.

\section{Data items}

The following information will be extracted from each article: author, journal title, year of publication, study setting, study design; demographic attributes such as age, sex, country, ethnicity; sample size, clinical features, investigations, modes and doses of treatment given, supportive care, outcome, strengths and limitations of the studies. The detailed data extraction form is available in the online supplemental file 2 .

\section{Expected outcome(s)}

1. Epidemiology of disease.

2. Clinical presentation and aetiopathology of the disease.

3. Investigations required and modes of treatment used.

4. Clinical outcomes of disease such as hospitalised, transferred to other facility, discharged alive, death, ICU admissions and/or left against medical advice.

\section{Risk of bias in individual studies}

Risk of bias will be independently assessed by two reviewers. All studies included for this review will be 
assessed. We will evaluate risk of bias for observational studies using 'Strengthening the Reporting of Observational Studies in Epidemiology' (STROBE) guidelines. ${ }^{16}$ A simple pro forma having three domains for assessing selection bias, information bias and confounding will be used. Information bias will include both differential misclassification and non-differential misclassification. Each of the three domains will be marked as either 'Yes' or 'No' for risk of bias. One point for each of the STROBE item will be given and study will be included for review if it has a minimum 12 quality score out of possible 23. Any differences between the two reviewers will be sorted after consulting the third reviewer or having a tie-breaker.

\section{Data synthesis}

A descriptive and quantitative synthesis will be used to summarise the results and designs of existing studies. First of all, a range of different clinical and epidemiological features and management done will be presented as narrative synthesis. We will combine the number of cases with individual epidemiological and clinical characteristics, aetiopathology, investigations required and the treatment modes used for each study, and calculate the combined percentage of individual clinical symptoms with $95 \%$ CI. The outcome of each case such as mortality, ICU admissions and complications will be reported in the final report in $\mathrm{n} \%$. Subgroups can be formed based on sex and ethnicity, which will be analysed if data permits.

We will try to synthesise the data, even if only two articles are reported.

\section{ETHICS AND DISSEMINATION}

This is a literature-based study with no ethical concerns. The data will be obtained from published/grey literature. Individual patient data are not required, hence, eliminating privacy concerns. The results of this study will be published in a peer-reviewed journal and presented at a conference.

\section{Patient and public involvement}

Patients were not involved during any of the stages of this protocol as it is a synthesis of available published data.

\section{DISCUSSION}

This systematic review will focus on summarising the clinical and epidemiological features of a novel challenge faced by the healthcare workers during COVID-19 pandemic. It will generate evidence for assisting in management of disease including laboratory investigations and treatment regimens being used. There is an urgent need to categorise this syndrome but lack of standardised data does not permit this. Our systematic review, being rapid and living, will help in overcoming this short fall, although robust data collection mechanisms are being developed and advocated worldwide.
This systematic review will follow quality standards as laid in the protocol, hence, generating the best possible evidence. The information compiled on the clinical features, along with outcomes of the disease, will add to the scarce data giving a new direction to the healthcare professionals as well as researchers worldwide.

Contributors All authors (AS, SN, PD, SK and SP) contributed to the development of the search strategy, inclusion/exclusion criteria and data extraction form. The protocol was drafted by AS and reviewed and edited by SN, PD, SK and SP. All authors have approved the final manuscript for submission.

Funding The authors have not declared a specific grant for this research from any funding agency in the public, commercial or not-for-profit sectors.

Competing interests None declared.

Patient consent for publication Not required.

Provenance and peer review Not commissioned; externally peer reviewed.

Supplemental material This content has been supplied by the author(s). It has not been vetted by BMJ Publishing Group Limited (BMJ) and may not have been peer-reviewed. Any opinions or recommendations discussed are solely those of the author(s) and are not endorsed by BMJ. BMJ disclaims all liability and responsibility arising from any reliance placed on the content. Where the content includes any translated material, BMJ does not warrant the accuracy and reliability of the translations (including but not limited to local regulations, clinical guidelines, terminology, drug names and drug dosages), and is not responsible for any error and/or omissions arising from translation and adaptation or otherwise.

Open access This is an open access article distributed in accordance with the Creative Commons Attribution Non Commercial (CC BY-NC 4.0) license, which permits others to distribute, remix, adapt, build upon this work non-commercially, and license their derivative works on different terms, provided the original work is properly cited, appropriate credit is given, any changes made indicated, and the use is non-commercial. See: http://creativecommons.org/licenses/by-nc/4.0/.

ORCID iD

Abhinav Sinha http://orcid.org/0000-0001-7702-3671

\section{REFERENCES}

1 Grasselli G, Pesenti A, Cecconi M. Critical care utilization for the COVID-19 outbreak in Lombardy, Italy. JAMA 2020;323:1545 https:// jamanetwork.com/journals/jama/fullarticle/2763188

2 Gorbalenya AE. Severe acute respiratory syndrome-related coronavirus - the species and its viruses, a statement of the coronavirus Study Group. bioRxiv 2020.

3 Sjödin $\mathrm{H}$, Wilder-Smith A, Osman S, et al. Only strict quarantine measures can curb the coronavirus disease (COVID-19) outbreak in Italy, 2020. Eurosurveillance 2020;25:2000280 https://www. eurosurveillance.org/content/

4 Pan A, Liu L, Wang C, et al. Association of public health interventions with the epidemiology of the COVID-19 outbreak in Wuhan, China. JAMA 2020;323:1915 https://jamanetwork.com/journals/jama/ fullarticle/2764658

5 Morand A, Urbina D, Fabre A. COVID-19 and Kawasaki like disease: the known-known, the unknown-known and the unknown, 2020.

6 Sandhaus H, Crosby D, Sharma A, et al. Association between COVID-19 and Kawasaki disease: vigilance required from Otolaryngologists. Otolaryngol Neck Surg 2020;019459982093023 http://journals.sagepub.com/doi/

7 Qiu H, Wu J, Hong L, et al. Clinical and epidemiological features of 36 children with coronavirus disease 2019 (COVID-19) in Zhejiang, China: an observational cohort study. Lancet Infect Dis 2020.

8 Richardson S, Hirsch JS, Narasimhan M, et al. Presenting characteristics, comorbidities, and outcomes among 5700 patients hospitalized with COVID-19 in the new York City area. JAMA 2020;323:2052 https://jamanetwork.com/journals/jama/fullarticle/ 2765184

9 Explained: Inflammatory syndrome in children possibly linked to COVID-19 | Euronews [online]. Available: https://www.euronews.com/ 2020/05/24/coronavirus-what-is-kawasaki-disease-and-its-possiblelink-with-covid-19-in-children [Accessed 26 May 2020].

10 Coronavirus (COVID-19) events as they happen [online]. Available: https://www.who.int/emergencies/diseases/novel-coronavirus-2019/ events-as-they-happen [Accessed 26 May 2020]. 
11 Verdoni L, Mazza A, Gervasoni A, et al. An outbreak of severe Kawasaki-like disease at the Italian epicentre of the SARS-CoV-2 epidemic: an observational cohort study. Lancet 2020;395:1771-8 https://linkinghub.elsevier.com/retrieve/pii/S014067362031103X

12 Riphagen S, Gomez X, Gonzalez-Martinez C, et al. Hyperinflammatory shock in children during COVID-19 pandemic. Lancet 2020;395:1607-8 http://www.ncbi.nlm.nih.gov/pubmed/ 32386565

13 Multisystem inflammatory syndrome in children and adolescents temporally related to COVID-19 [online]. Available: https://www. who.int/news-room/commentaries/detail/multisystem-inflammatorysyndrome-in-children-and-adolescents-with-covid-19 [Accessed 1 Jun 2020].
14 Shamseer L, Moher D, Clarke M, et al. Preferred reporting items for systematic review and meta-analysis protocols (PRISMA-P) 2015: elaboration and explanation. BMJ 2015;349:g7647.

15 A rapid living systematic review of clinico-epidemiological characteristics of Kawasaki disease in COVID-19 pediatric patients [online]. Available: https://www.crd.york.ac.uk/prospero/display_ record.php?ID=CRD42020187427 [Accessed 26 May 2020].

16 von Elm E, Altman DG, Egger M, et al. The strengthening the reporting of observational studies in epidemiology (STROBE) statement: guidelines for reporting observational studies. Int J Surg 2014;12:1495-9. 